\title{
Sufu regulation of Hedgehog signaling in P19 cells is required for proper glial cell differentiation
}

\author{
Danielle M. Spice ${ }^{1,2}$, Joshua Dierolf ${ }^{3}$ and Gregory M. Kelly ${ }^{1-3, *}$ \\ Molecular Genetics Unit, Department of Biology, Western University, London, ON, Canada N6A5B7 \\ 2 Children's Health Research Institute, London, ON, Canada \\ Department of Physiology and Pharmacology, Western University, London, ON, Canada N6A5B7
}

\begin{abstract}
Hedgehog signaling is essential for vertebrate development, however, less is known about the negative regulators that influence this pathway during the differentiation of cell fates. Using the mouse P19 embryonal carcinoma cell model, Suppressor of Fused (SUFU), a negative regulator of the Hedgehog pathway, was investigated during retinoic acid-induced neural differentiation. We found Hedgehog signaling was activated in the early phase of neural differentiation and became inactive during terminal differentiation of neurons and astrocytes. SUFU, which regulates signaling at the level of GLI, remained relatively unchanged during the differentiation process, however SUFU loss through CRISPIR-Cas9 gene editing resulted in decreased cell proliferation and ectopic expression of Hedgehog target genes. Interestingly, SUFU-deficient cells were unable to differentiate in the absence of retinoic acid, but when differentiated in its presence they showed delayed and decreased astrocyte differentiation; neuron differentiation did not appear to be affected. Retinoic acid-induced differentiation also caused ectopic activation of Hh target genes in SUFU-deficient cells and while the absence of the GLI3 transcriptional inhibitor suggested the pathway was active, no fulllength GLI3 was detected even though the message encoding Gli3 was present. Thus, the study would indicate the proper timing and proportion of glial cell differentiation requires SUFU, and its normal regulation of GLI3 to maintain Hh signaling in an inactive state.
\end{abstract}

\section{Introduction}

Hedgehog (Hh) signaling plays pivotal roles in vertebrate development, and its deregulation leads to birth defects and/or cancer progression. In normal neural development Sonic Hedgehog (SHH) is essential in patterning the differentiation of motor neurons and interneurons in the developing neural tube [1] and it induces the differentiation of cerebellar neurons and glial cells [2]. When canonical signaling is off and the Hh ligand is absent, the transmembrane receptor Patched (PTCH) inhibits the transmembrane protein Smoothened (SMO) from translocating to the plasma membrane of the primary cilium [3]. This inhibition allows the scaffolding protein Suppressor of Fused (SUFU) to sequester full length GLI transcription factors in the cytoplasm [4] or promotes GLI phosphorylation [5] and subsequent partial proteosomal degradation into a truncated repressor [6, 7]. In the presence of Hh, however, PTCH is bound and relieves the inhibition of SMO. This allows the SUFU-GLI complex to be recruited into the primary cilium [8] where SUFU dissociates from GLI allowing the translocation of GLI to the nucleus where it acts as a transcriptional activator [7]. In vertebrates there are three GLI transcription factors, GLI1, GLI2 and GLI3. GLI1 acts solely as an activator, however, as a Hh target gene its presence appears only after Hh activation [6]. GLI3 acts primarily as a transcriptional repressor, although both GLI2 and GLI3 can act as either activators or repressors [6]. Thus, while Hh target gene transcription is tightly regulated through GLI, the latter are also regulated by other proteins.

SUFU plays an essential role in regulating GLI processing, and thus is key to Hh pathway activation. Sufu loss of function alleles result in the constitutive expression of $\mathrm{Hh}$ target genes in the absence of a Hh ligand [9, 10], through the reduced processing and stability of the GLI3 repressor [11]. Overactivation of Hh signaling through Sufu loss in humans causes medulloblastoma tumor growth $[12,13]$ and Nevoid Basal Cell Carcinoma Syndrome [13, 14], while mouse embryos containing a targeted deletion of Sufu 
are embryonic lethal at E9.5 due to neural tube closure defects [10]. Furthermore, the specific loss of Sufu function in the cerebellum results in developmental delays in neuron differentiation [15] and cerebellum mispatterning [16].

In the present study an in vitro system utilizing the mouse P19 embryonal carcinoma cell line was employed to study when the Hedgehog pathway is activated and the effects of loss of Sufu function on this activation. The cell line, derived from the resulting teratoma of the subcutaneous implantation of a E7.5 mouse embryo into the testis of another mouse [as reviewed in 17] are readily differentiated into neural lineages in the presence of retinoic acid (RA) [18]. RA-induced differentiation of P19 cells forms functional neurons and supporting astrocytes [19], and although this is well-known, few studies have investigated the role of Hh signaling in this system [20,21]. Of these, the role of Gli2 has been reported, but where one study shows the knockdown of Gli2 promotes neuronal differentiation [21], another reveals that the overexpression of a constitutively active Gli2 causes the same phenotype [20]. Even though Hh signaling is precisely controlled in neural cell fate decisions in vivo, and that this is mediated through negative regulators, what remains to be resolved is how SUFU is involved in neuronal and glial cell differentiation. To address this shortfall, this study aims to better understand the essential role of SUFU in neuroectodermal differentiation. Using the P19 cell model and timeline investigations, we show when Hh signaling is activated during RA-induced neural differentiation and through genetic ablation of Sufu demonstrate that there is an essential role of SUFU in glial cell fate determination.

\section{Materials and Methods}

\subsection{Cell culture \& Differentiation}

P19 embryonal carcinoma cells (a gift from Dr. Lisa Hoffman, Lawson Health Research Institute, London, ON, Canada) and Sufu knockout (Sufu- ${ }^{-}$, described below) P19 cells were cultured on adherent tissue culture plates in DMEM containing 5\% fetal bovine serum (FBS) and $1 \%$ pen/strep at $37^{\circ} \mathrm{C}$ and $5 \% \mathrm{CO}_{2}$. For passaging, cells were trypsinized (TrypLE Express), centrifuged and re-suspended as single cells every 4 days or at $70 \%$ confluency, whichever occurred first.

Differentiation of P19 cells was adapted from McBurney et al. 1988 [19]. To induce neural differentiation, approximately $1.05 \times 10^{6}$ cells were cultured on bacterial grade Petri dishes for 4 days in $0.5 \mu \mathrm{M}$ RA to form embryoid bodies. After this incubation, aggregates were subsequently plated on adherent tissue culture dishes in the presence of $0.5 \mu \mathrm{M} \mathrm{RA}$, for a total of 10,14 or 17 days. Untreated controls were grown for 4 days on bacterial grade Petri dishes and re-plated on adherent tissue culture dishes for the total number of days stated above.

\subsection{Cas9 plasmid preparation}

The pSpCas9(BB)-2A-Puro (PX459) V2.0 (Addgene plasmid 62988; http://n2t/addgene:62988) was used, where sgRNAs for Sufu (Supplementary Table 1) were cloned into the PX459 vector using the protocol of Ran et al. 2013 [22]. Briefly, sgRNAs were amplified and phosphorylated, the vector digested using the BbsI restriction enzyme, and vector and sgRNA were incubated at room temperature for $1.5 \mathrm{~h}$ for ligation. Ligated plasmids were transformed into competent DH5alpha bacteria and grown on ampicillin containing agar plates. Colonies were selected and isolated plasmids were sent for sequencing at the London Regional Genomics Centre (Robarts Research Institute, London, ON) using the U6 primer (Supplementary Table 1).

\subsection{Knockout lines}

P19 cells were incubated with $2 \mu \mathrm{g}$ of PX459-sgRNA plasmid in $10 \mu \mathrm{L}$ of Lipofectamine 2000 (Invitrogen). Media was changed after $4 \mathrm{~h}$, and cells were grown to $70 \%$ confluency and then passaged as single cells in a 96 well plate and incubated with $1 \mu \mathrm{g} / \mathrm{mL}$ puromycin. Media containing $1 \mu \mathrm{g} / \mathrm{mL}$ puromycin was replaced every $24 \mathrm{~h}$ for 4 days, and then cells were grown in complete media without puromycin until ready to be passaged. Knockout genotypes were determined by collecting genomic DNA (Qiagen DNeasy® Blood \& Tissue kit, 69504), and performing standard PCR (DreamTaq Master Mix (2X), Thermo Scientific, K1081) with Sufu-specific primers (Supplementary Table 1) and a Vapo.protect Thermocycler (Eppendorf). Amplicons were sequenced at the London Regional Genomics Centre and compared to wildtype sequences using pair-wise sequence alignment (Geneious 2021 ${ }^{\mathrm{TM}}$ ). KO line \#2 sequencing was examined using TIDE analysis (http://shinyapps.datacurators.nl/tide/) [23] to further 
investigate the nature of the allele. TIDE analysis was unable to interrogate KO line \#1 as the length of deletion was outside of the tool parameters.

\subsection{Real-time reverse transcriptase PCR}

To determine relative mRNA expression levels total RNA was collected from differentiated P19 cells after 1, 2, 3, 4, 6, 8, 10, 14 and 17 days after initial treatment (where cells collected between days 1-4 were in aggregates and those collected between days 6-17 were re-plated cells) using QiaShredder (Qiagen, 79654) and RNeasy (Qiagen, 74104) kits. RNA was reverse transcribed into cDNA using a High-Capacity cDNA Reverse Transcription Kit (Thermo Fisher Scientific, 4368814). Primers were designed for: L14, Shh, Ihh, Dhh, Smo, Ptch1, Ptch2, Sufu, Gli1, Gli2, Gli3, Ascl1, NeuroD1, NeuroG1, and Nestin (Supplementary Table 1). RT-qPCR was conducted according to the following reaction conditions: $500 \mathrm{nM}$ of each reverse and forward primers, $10 \mu \mathrm{L}$ of SensiFAST SYBR No-ROX Mix (Bioline, FroggaBio, BIO-98050), and $1 \mu \mathrm{L}$ of cDNA template. Samples were analyzed using a CFX Connect Real-Time PCR Detection System (Bio-Rad) using the comparative cycle threshold method (2- $\left.{ }^{-\Delta \Delta C t}\right)$. Relative mRNA expression was normalized to L14 expression and were subsequently normalized to untreated or wildtype untreated controls to determine fold change expression.

\subsection{Immunoblot Analysis}

Cells were lysed in $150-500 \mu \mathrm{L}$ of $2 \%$ sodium dodecyl sulfate buffer containing $62.5 \mathrm{mM}$ Tris-HCL pH 6.8, 10\% glycerol, 5\% mercapto-2-ethanol and 1X Halt Protease Inhibitor Cocktail (Thermo Scientific, 1862209) at a 1:200 ratio. Protein lysates were sonicated for $30 \mathrm{sec}$ on ice and protein concentrations were measured using the DCTM Protein Assay (Bio-Rad, 5000113). Approximately $10-20 \mu \mathrm{g}$ of protein were separated on denaturing 6-10\% polyacrylamide gels for approximately $90 \mathrm{~min}$ at $120 \mathrm{~V}$, then transferred to PVDF membranes (Bio-Rad, 1620177) at $20 \mathrm{~V}$ overnight at $4^{\circ} \mathrm{C}$. Membranes were incubated for $1 \mathrm{~h}$ at room temperature in Tris-buffered saline with $0.1 \%$ Tween-20 (TBS-T) containing 5\% skim milk, and then probed with primary antibodies to $\beta$-III-tubulin (TUJ1, 1:1000; Cell Signaling Technology, 5568), GLI3 (1:1000; R\&D Systems, AF3690), SUFU (1:1000; abcam, ab28083), GFAP (1:1000; Invitrogen, 14-9892-80), Nestin (1:1000; Cell Signaling Technology, 4760), and $\beta$-actin (1:10,000; Santa Cruz Biotechnology, sc-47778) overnight at $4^{\circ} \mathrm{C}$. Membranes were washed 3 times for 5 min with 1X TBS-T and were probed with the appropriate HRP-conjugated secondary antibodies (1:10,000, Sigma) for $2 \mathrm{~h}$ at room temperature. After washing 3 times for 5 min with $1 \mathrm{X}$ TBS-T, signals were detected using the Immobilon ${ }^{\circledR}$ Classico Western HRP Substrate (Millipore, WBLUC0500) and were imaged using a Chemi Doc Touch System (Bio-Rad).

\subsection{Immunofluorescence}

P19 wildtype and $S u f u^{-/}$cells were differentiated as stated previously, on coverslips coated with poly-L-lysine hydrobromide (Sigma, P5899). Coverslips were washed 3 times with PBS before they were used for cell culture. After differentiation, cells were washed for 1 min with PBS before fixation with 4\% paraformaldehyde in PBS for 10 min at room temperature. Cells were washed 3 times for $5 \mathrm{~min}$ with ice-cold PBS before being permeabilized in $0.2 \%$ Triton-X-100 in PBS for $10 \mathrm{~min}$ at room temperature, then washed 3 times for $5 \mathrm{~min}$ before blocking for $30 \mathrm{~min}$ at room temperature in $1 \%$ Bovine Serum Albumin, $22.52 \mathrm{mg} / \mathrm{mL}$ glycine and $0.1 \%$ Tween-20 in PBS. Coverslips were incubated overnight in a humidity chamber with $\beta$ III-tubulin (TUJ1, 1:400; Cell Signaling Technology, 5568) and GFAP (1:500; Invitrogen, 14-9892-80) in blocking solution. Coverslips were then washed 3 times for 5 min with PBS before cells were incubated in secondary antibodies, Alexa Fluor ${ }^{\mathrm{TM}} 660$ goat anti-mouse IgG (Invitrogen, A21054) and Goat anti-Rabbit IgG Alexa Fluor Plus 488 (Invitrogen, A32731TR) in blocking solution in the dark for $1 \mathrm{~h}$ at room temperature. Coverslips were washed 3 times for 5 min with PBS in dark conditions before being mounted onto microscope slides using Slowfade ${ }^{\mathrm{TM}}$ Gold antifade reagent with DAPI (Invitrogen, S36942). Coverslips were sealed to slides using nail polish then imaged using a Zeiss AxioImager Z1 Compound Fluorescence Microscope. All immunofluorescence images were taken at the Integrated Microscopy Facility, Biotron (Western University, London, ON, Canada). 
P19 wildtype and Sufuc- cells were differentiated as stated previously. Cells were washed three times with ice cold PBS wihout magnesium or chloride (PBS(-/-)) (Thermo Fisher A1286301) and dissociated with 0.25\% Trypsin. Subsequent PBS(-/-) washes were completed 3 times, 5 minutes each. Trypsin was deactivated with DMEM/F12 (ThermoFisher 11320033) containing 10\% ES-grade Fetal Bovin Serum (FBS) (Thermo Fisher 10439016), and then cells were centrifuged at $300 \mathrm{~g}$ for $5 \mathrm{minutes}$ and reconstituted in PBS(-/-). Cells were counted using a trypan blue assay, separated to approximately $1.0 \times 10^{6}$ cells per tube, washed in Flow Cytometry Staining Buffer (FCSB(10\% FBS in PBS(-/-)),-centrifuged as previously described and the buffer aspirated. Cells were fixed using 4\% formaldehyde (Electron Microscopy Sciences 15680) in PBS(-/-) for 10 min at room temperature, washed with ice cold PBS and then permeabilized with $0.2 \%$ Triton-X-100 in PBS(-/-) for 10 min at room temperature before subsequently washing with PBS(-/-). Cells were incubated with $1 \mu \mathrm{L}$ of GFAP Monoclonal Antibody (GA5), Alexa Fluor 488, eBioscience ${ }^{\mathrm{TM}}$ (Invitrogen, 53-9892-82) per million cells for $1 \mathrm{~h}$ at room temperature, and then washed with PBS (-/-), strained using a $40 \mu \mathrm{m}$ cell strainer (Falcon ${ }^{\mathrm{TM}}$ C352340) and analyzed using a BD FACSCanto II flow cytometer at the London Regional Flow Cytometry Facility (Robarts Research Institute). Data were analyzed using FlowJo (BD).

\section{Results}

3.1 Retinoic acid and cell aggregation induces P19 neural differentiation

Neuronal differentiation was induced by culturing cells for 4 days in the presence of $0.5 \mu \mathrm{M}$ RA to form cell aggregates that were then plated for an additional 6 days to form neurons (Figure 1A). RT-qPCR was used to test for neural differentiation, and as expected NeuroG1 showed increased expression between days 0-6 with a subsequent decline on days 8-10 (Figure 1B). NeuroD1 and Ascll both increased in expression throughout RA treatment to days 6-10 when neurons are forming (Figure 1C and D, respectively). Neural differentiation in the presence of RA was confirmed through immunoblot analysis where NESTIN, a marker of neuronal precursors, showed increased intensity at days 6 and 10 (Figure 1E and Figure 1F). Nestin gene expression, however, showed no change through differentiation in RA treated cells (Figure 1G). $\beta$-III-tubulin, a neuronal marker, was only detected following RA treatment, and only after 10 days (Figure 1E). Together, these results confirmed that our differentiation protocol was successful at differentiating neural precursors and neurons. 
A

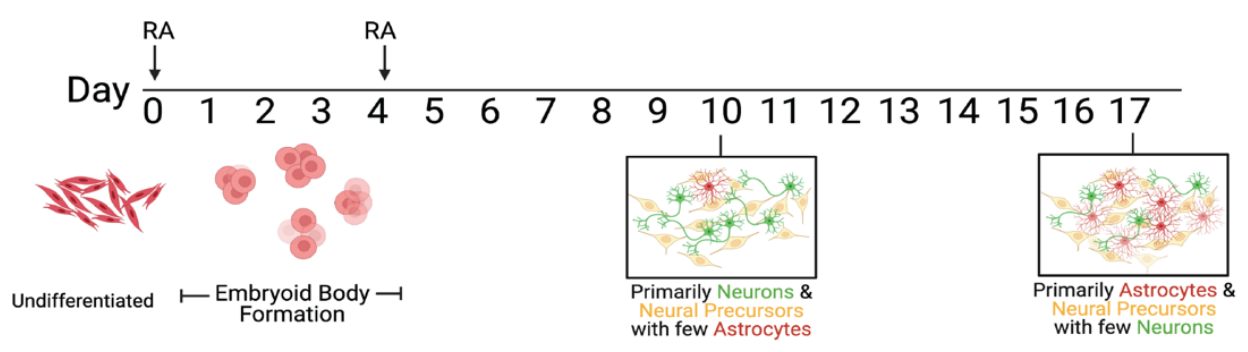

B

C
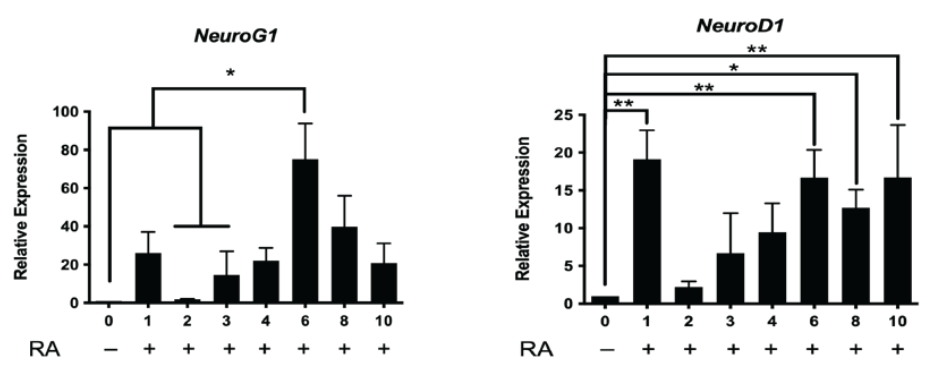

D

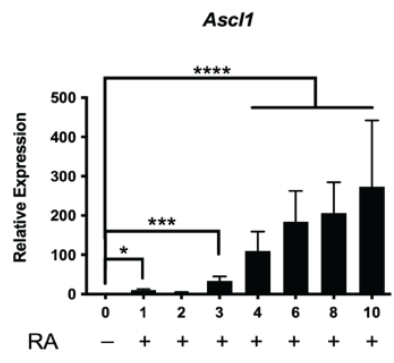

$E$

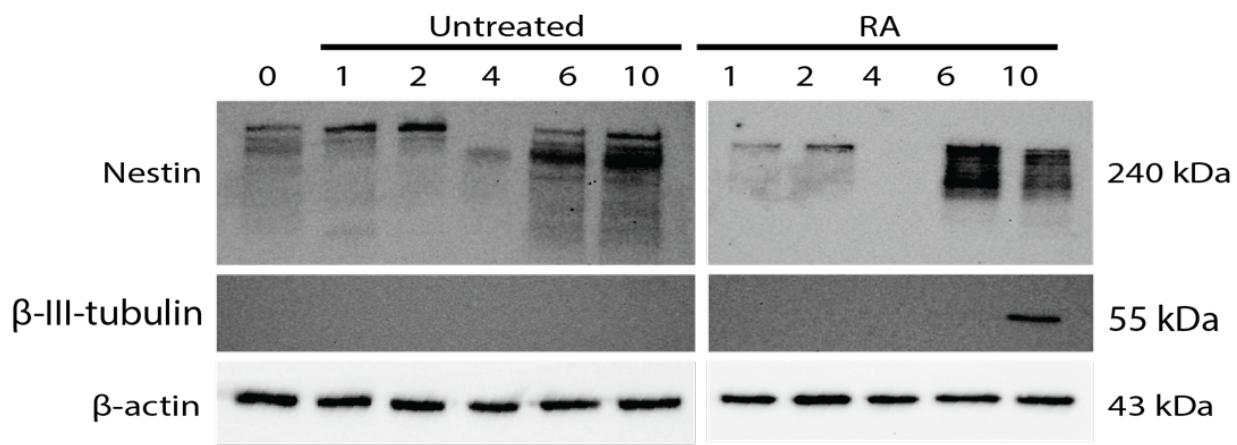

$\mathrm{F}$

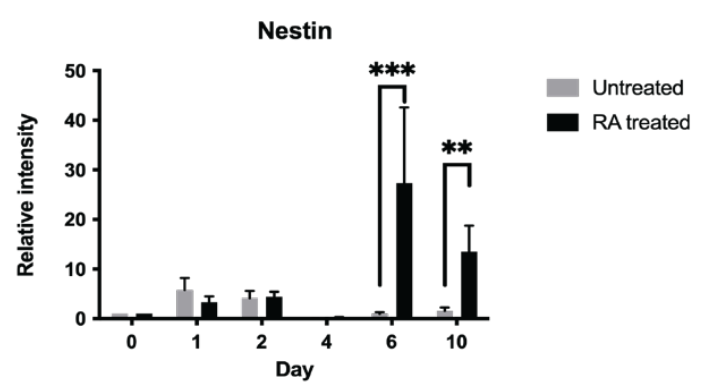

G

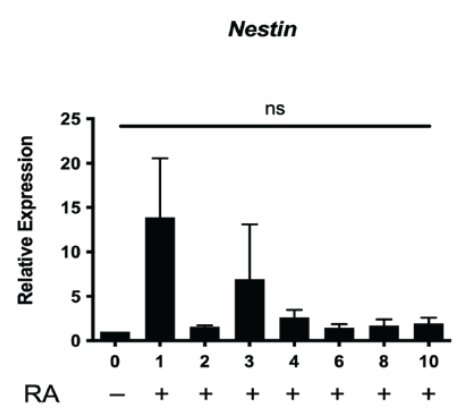

Figure 1 - Retinoic acid is required for P19 cell neuronal differentiation. A) Schematic of in vitro differentiation of P19 cells toward neural lineages. B) NeuroG1, C) NeuroD1, and D) Ascl1 expression on days 0-10 of differentiation. E) Immunoblot of neural precursor marker Nestin and neuron marker $\beta$-III-tubulin over the same period. F) Densitometric analysis of Nestin intensity from E, and Nestin gene expression (G) during differentiation. ${ }^{*} P<0.05, * * P<0.01,{ }^{*} * * P<0.001,{ }^{*} * * * P<0.0001$. Panel A was created using BioRender.com.

3.2 Hh signaling is activated early in neuronal differentiation

Since numerous reports have shown that Shh signaling is vital in vertebrate neural differentiation [as reviewed in 1-3], Hh expression must be present during RA-induced P19 cell neural differentiation. The relative expression of vertebrate Hedgehogs and the canonical Hh pathway components were explored using RT-qPCR between days 0-10 of RA treatment. Expression of Hh ligands Dhh, Ihh, and Shh (Figure 2A-C), Hh pathway components Ptch1, Ptch2, Smo, Gli2, Gli3 (Supplemental Figure 1), and Hh target 
gene Glil (Supplemental Figure 1D) and Sufu (Figure 4A) was confirmed during days 0-10 of RA treatment. Dhh, Ihh and Shh showed increased expression 1 day after RA induction (Figure 2A-C, respectively) and this coincided with increased expression of Gli1 (Supplemental Figure 1D). The presence and activity of GLI transcription factors are necessary for Hh signal activation [3], where GLI3 is key for early signal modulation, serving as either an activator or repressor [6]. Thus, the ratio between GLI3 fulllength (GLI3FL) to GLI3 repressor (GLI3R) protein is an important indicator of how GLI3 is acting in the Hh pathway. When GLI3 was examined after 1 day in culture, results showed that the GLI3FL:GLI3R ratio increased in untreated and RA-treated cells (Figure 2D and E). Thus, it would appear the Hh pathway is activated early in the neural differentiation protocol, leading to GLI3 activation. This ratio would decrease, showing GLI3R increased as cells differentiated (Figure 2D and E).

\subsection{Hh signaling is not activated during astrocyte differentiation}

Culturing P19 cells for 4 days in the presence of $0.5 \mu \mathrm{M}$ RA to form cell aggregates that were then plated for an additional 13 days induced astrocyte differentiation (Figure 1A). $\beta$-III-tubulin signals were seen on day 10 (Figure 3A), like previously shown (Figure 1E), and astrocyte differentiation was confirmed by the presence of glial fibrillary acidic protein (GFAP) at days 10, 14 and 17 of RA treatment (Figure 3A). Hedgehog signal activation was again determined using the GLI3FL:GLI3R ratio in control and RA-treated cells (Figure 3B). Results show that at these later stages of differentiation there were no significant differences in the GLI3FL:GLI3R ratio (Figure 3C), suggesting that Hh signaling was inactive during astrocyte differentiation.

A
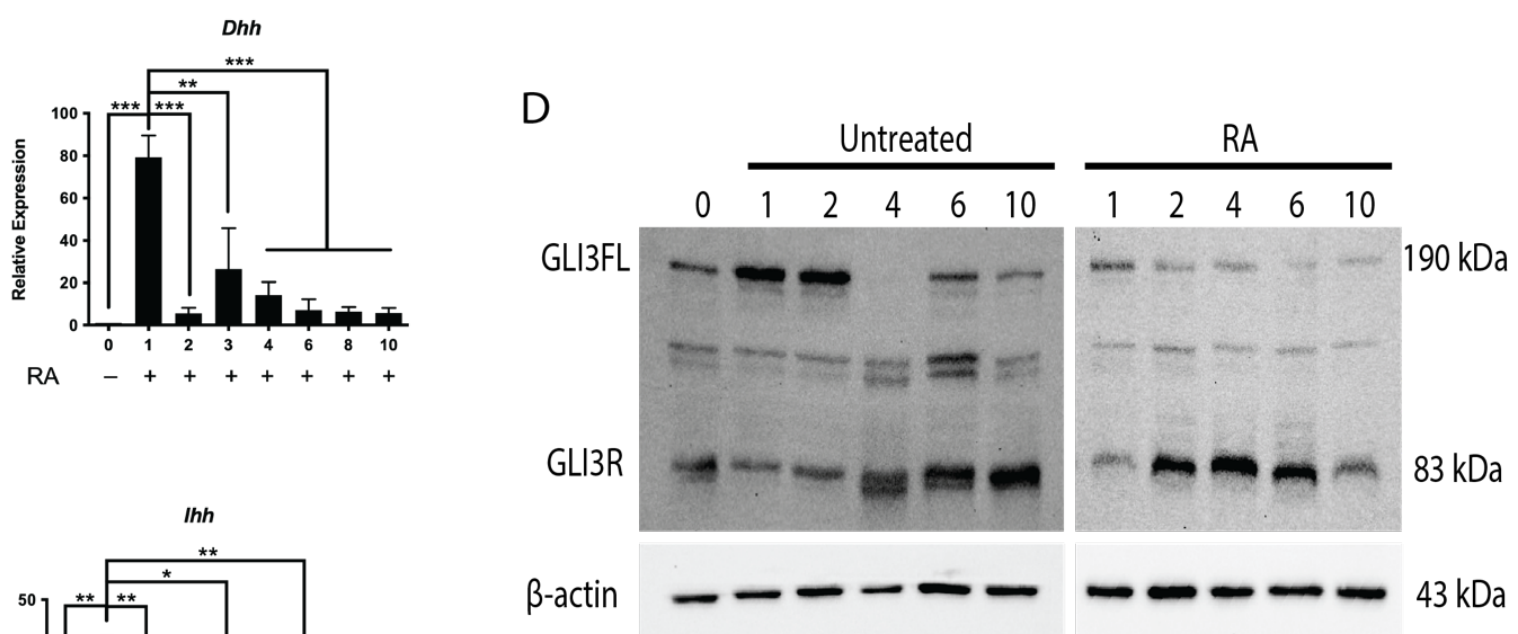

B

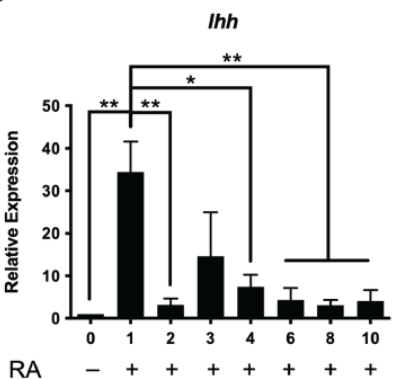

E

C Shh
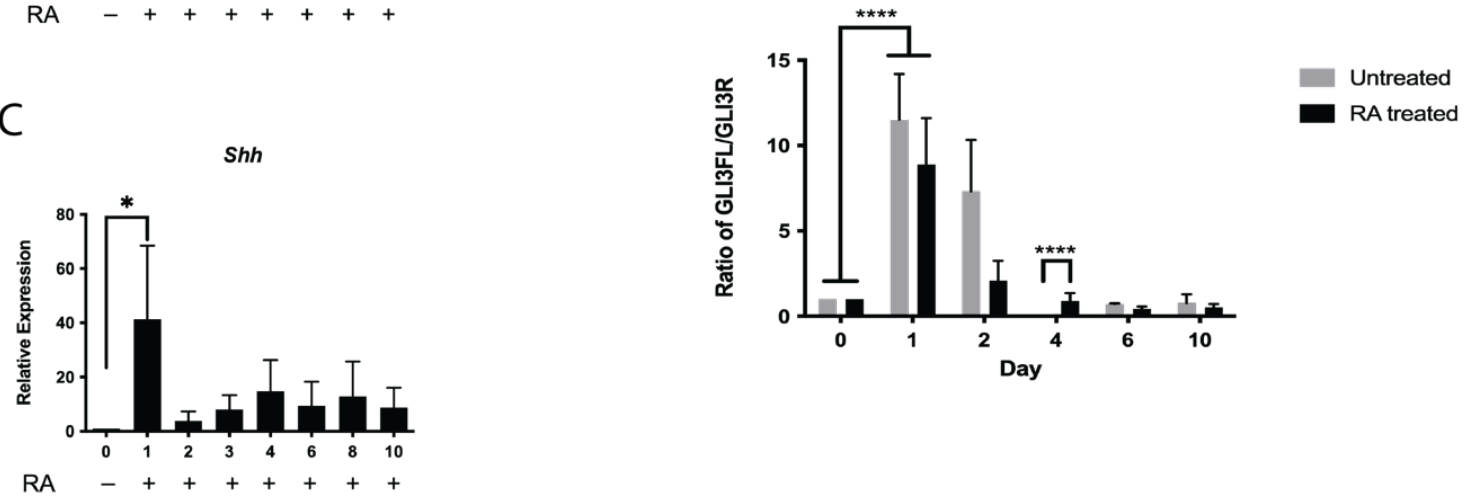

Figure 2 - Hh signaling is activated early in RA-induced differentiation. Expression of Hh ligands A) $D h h$, B) $I h h$, and C) Shh, and D) immunoblot of GLI3 full length (GLI3FL) and GLI3 repressor (GLI3R) on days 0-10. E) Densitometric analysis of GLI3FL to GLI3R ratio from previous immunoblot. $* P<0.05$, **P<0.01, ***P<0.001, ****P<0.0001. 
A
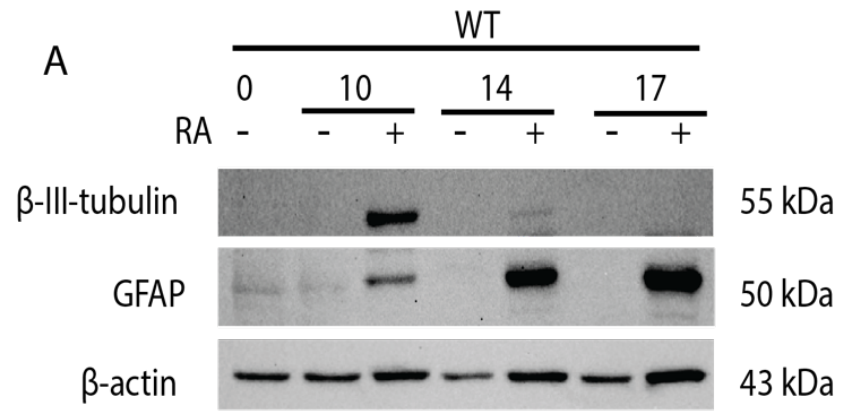

B

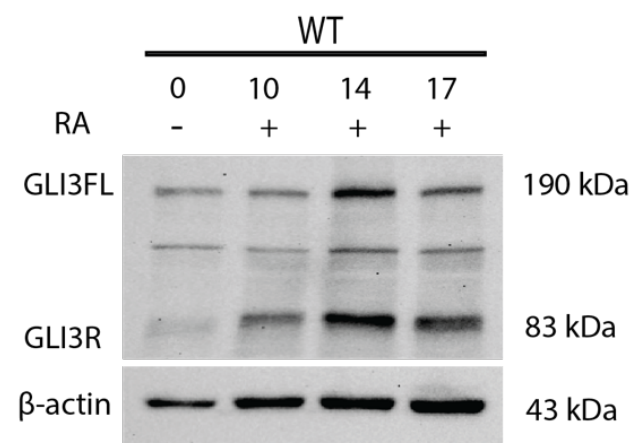

C

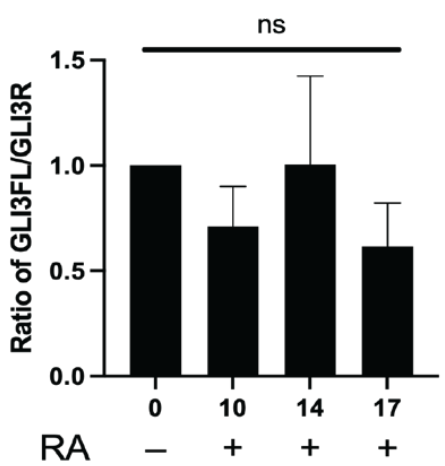

Figure 3 - RA-induced differentiation on days 10-17 favors astrocyte formation. A) Immunoblot of neuron ( $\beta$-III-tubulin) and astrocyte marker (glial fibrillary acidic protein, GFAP) on days 0-17. B) Immunoblot of GLI3 full length (GLI3FL) and GLI3 repressor (GLI3R) during days 0-17. C) Densitometric analysis of the GLI3FL to GLI3R ratio of immunoblot in B.

\subsection{SUFU levels during neural differentiation}

Given the information, we report that Hh signaling triggers differentiation, but how the pathway is attenuated thereby allowing the terminal neuron and glial cell differentiation to occur remained to be determined. One gene of interest, Sufu, encodes a negative regulator of the pathway that inhibits GLI activity and as reported by Humke et al. [7], promotes GLI3 conversion to a repressor. During neuronal differentiation (days 0-10) Sufu gene expression increased at days 1, then later on days 8 and 10 of RA treatment (Figure 4A); however, at the protein level no significant changes in SUFU intensity between untreated or RA treated cells at days 0-10 was observed (Figure 4B), and this was confirmed after densitometric analysis (Figure 4C). During astrocyte differentiation (day 10-17) Sufu expression showed no apparent change relative to the control on day 0 (Figure 4D), while SUFU protein intensity decreased significantly at the later stages (Figure 4E and F). That Sufu expression remain largely unchanged and only at later stages, coinciding with astrocyte differentiation, does the protein levels decrease, would suggest that SUFU is only needed during the early stages of differentiation. 
A

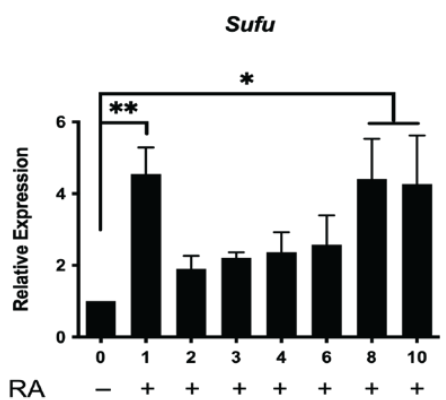

B

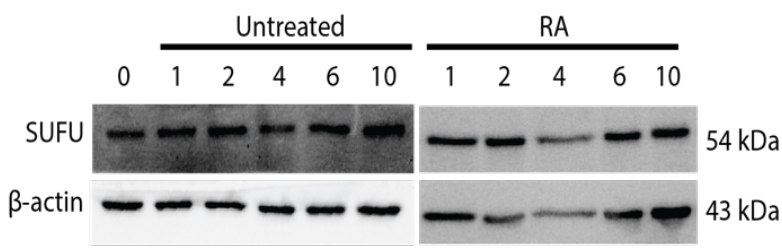

C

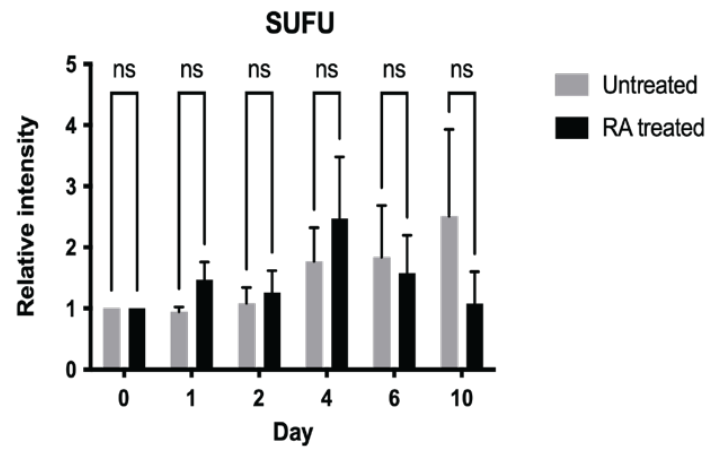

$\mathrm{D}$

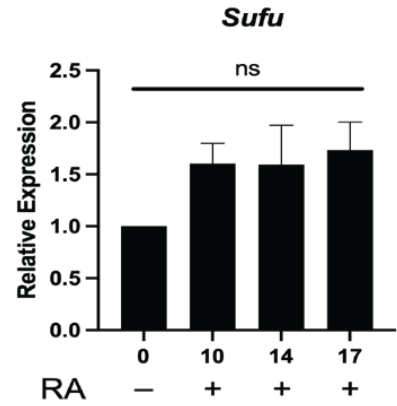

$E$

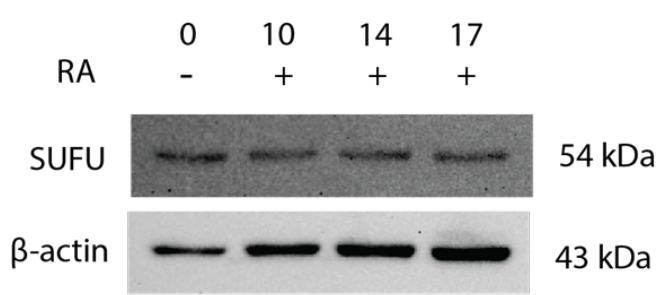

$\mathrm{F}$

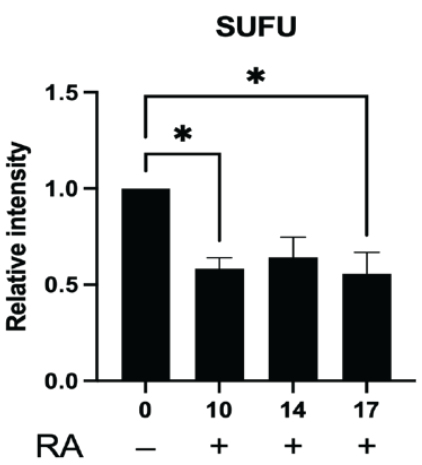

Figure 4 - SUFU levels during RA-induced differentiation. A) Expression of Sufu on days 0-10. B) Immunoblot of SUFU protein on days 0-10 and C) densitometric analysis of the immunoblot. D) Expression of Sufu on days 0-17, E) immunoblot analysis of the SUFU protein at these timepoints, and $\mathbf{F}$ ) densitometric analysis of the immunoblot in $\mathbf{E}$. $* P<0.05, * * P<0.01$.

\subsection{Sufu knockout activates Hedgehog signaling}

Aberrant SUFU activity is associated with tumorigenesis [12-14], and given that SUFU acts as an essential negative regulator of GLI3 [7], and GLI2 [4, 7], its loss would be expected to induce Hh signaling. To test this, CRISPR-Cas9 was used to target exon 6 of the Sufu gene (Figure 5A). Gene targeting resulted in the formation of two clones, one with an $81 \mathrm{bp}$ deletion (KO \#1) that spans the intron-exon boundary between intron 5 and exon 6. In silico analysis was performed for the predicted translation of the $81 \mathrm{bp}$ deletion and it revealed a premature stop codon at amino acid location 231 of the 484 amino acid SUFU polypeptide. The second mutation, KO \#2, revealed by TIDE analysis [23] of amplified genomic Sufu, showed a 1 bp insertion of a guanine residue just upstream of the PAM sequence, also resulting in a premature stop codon at amino acid location 246 of the polypeptide (Supplemental Figure 2). Immunoblot analyses using a SUFU antibody targeted to the C-terminal end of the polypeptide, a region previously reported to be required for GLI binding [4, 24], showed a signal in WT cells after 3 days in untreated monolayer culture; however, no signal was seen in either mutant under similar culturing conditions (Figure 5B).

CRISPR-Cas9-generated mutant $S u f u^{--}$alleles resulted in decreased cell numbers compared to WT cells cultured to $96 \mathrm{~h}$ (Figure 5C); however, the specific reason(s) for this remain unknown. Furthermore, compared to WT cells, KO Sufu lines \#1 or \#2 showed significantly increased Gli1, Ptchl, and Ascll gene expression in undifferentiated, monolayer cultures (Figure 5D). Together, the data showed the loss of SUFU affects the proliferation rate of undifferentiated cells, and it also aberrantly induced the activation of transcriptional targets of the Hh pathway. 


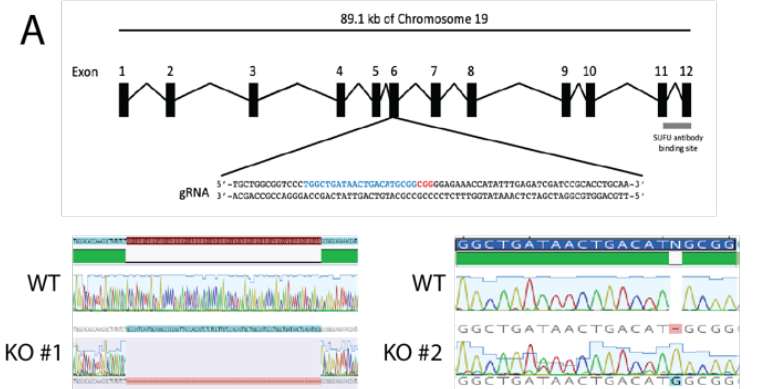

C

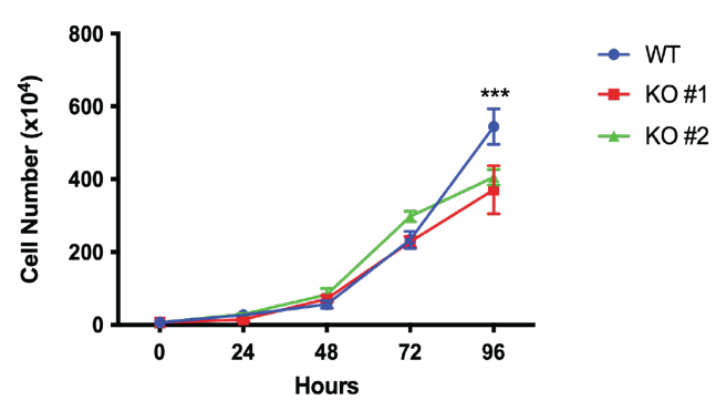

B

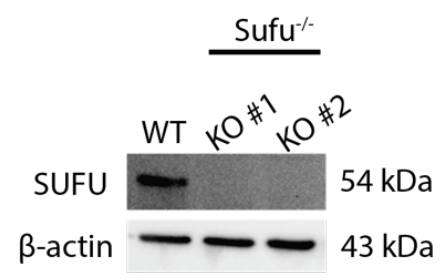

D

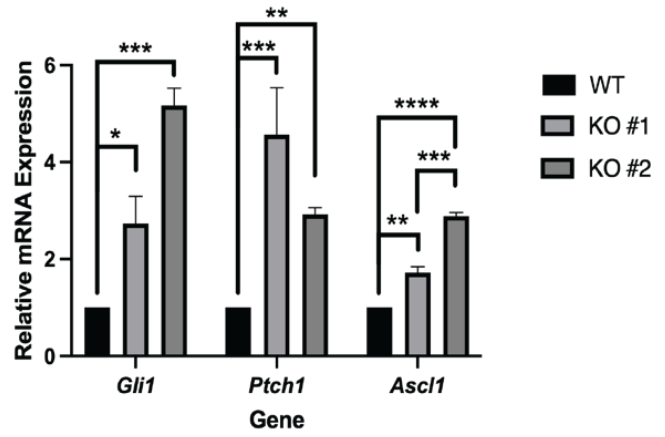

Figure 5 - CRISPR-Cas9 knock out of $S u f u$ activates Hh signaling in undifferentiated P19 cells. A) Schematic showing gRNA targeting exon 6 of the Sufu gene yielding two KO clones. B) SUFU levels in wildtype (WT) and Sufu $u^{-\leftarrow}$ clones, and C) cell count curves of WT and Sufu ${ }^{-/}$clones between 0-96 hours. D) Expression of Hh target genes Gli1, Ptch1 and Ascll in WT and Sufü clones on day 3 of undifferentiated cultures. $* P<0.05$, ** $P<0.01$, $* * * P<0.001$, ****P<0.0001.

\subsection{Sufu knockout delays and decreases glial cell fate}

After showing that WT cells induced Hh signaling after 1 day in culture (Figure 1B-D), and that Sufu $u^{--}$cells have ectopic Hh target gene expression (Figure 5D) we predicted that these cells would be either induced to differentiate in the absence of RA and/or they would induce neural differentiation earlier than that in WT cells. Results with the KO Sufu \#2 line showed $\beta$-III-tubulin and GFAP signals during the timepoints investigated (Figure 6A), but RA was required and no signals were detected in its absence. Similar results were seen with the other Sufu KO line (data not shown). Since Sufu $u^{-/}$cells were able to induce markers of differentiation and like WT cells this required RA, we compared both knockout lines to WT cells at days 10, 14 and 17 of RA treatment (Figure 6B) to see if the timing of differentiation was affected by the loss of SUFU. Results appeared to show $\beta$-III-tubulin levels decrease in WT and Sufu ${ }^{-/}$cells over time to day 17 of RA treatment (Figure 6B), but since densitometric analyses revealed no significant changes between cell lines (Figure 6C), then the presence or absence of Sufu must have no effect on neuron differentiation. Astrocyte differentiation was, however, affected and GFAP signals on blots were absent on day 10 of RA treatment in Sufu- cells (Figure 6B). Quantification could not be performed as GFAP signals were absent in the KO lines on day 10 (Figure 6D). Quantification results also confirmed GFAP levels were reduced significantly in the Sufu $u^{-/}$lines on day 14 (Figure 6D). Interestingly, there appeared to be a recovery of GFAP levels by day 17, and no significant differences in the relative intensity were detected by densitometry in WT or KO cells on that day (Figure 6D).

Immunofluorescence analyses and antibodies to $\beta$-III-tubulin and GFAP were used on WT and Sufu $u^{-\leftarrow}$ cells at day 17 of RA treatment to confirm the results that only astrocyte differentiation was affected in Sufu ${ }^{-/}$cells. Although expecting no differences in either marker between the two cell lines on day 17, as evident from immunoblots and their quantification (Figure 6B, C and D), immunofluorescence analysis revealed Sufü cells had fewer GFAP-positive cells (Figure 7A). To resolve this discrepancy, flow cytometry was performed on single cells and results showed WT cells having a significantly higher population ( $60-70 \%$ of live cells) of GFAP-positive cells compared to Sufu $u^{-/}$cells ( $\sim 6-25 \%$ of live cells) (Figure 7B). Statistical analysis on the flow data confirmed the immunofluorescence results and showed that there were fewer GFAP-positive cells in the Sufu $u^{-/}$population (Figure 7C). Together, this data supports the idea that the loss of Sufu does not alter neuronal differentiation as evident by the appearance of neurons labeled with $\beta$-III-tubulin, however, it does delay and decrease astrocyte differentiation. 
A

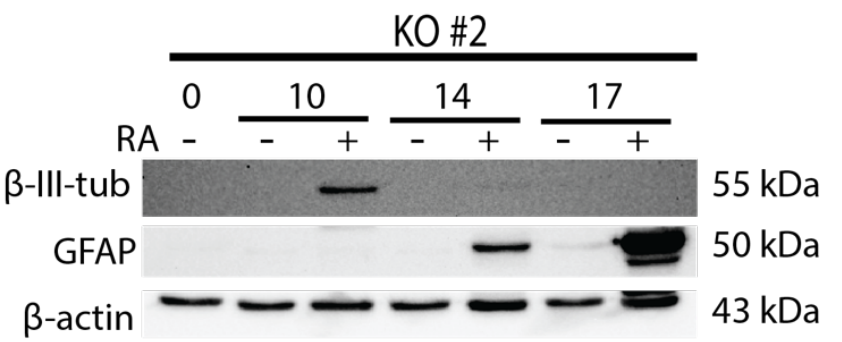
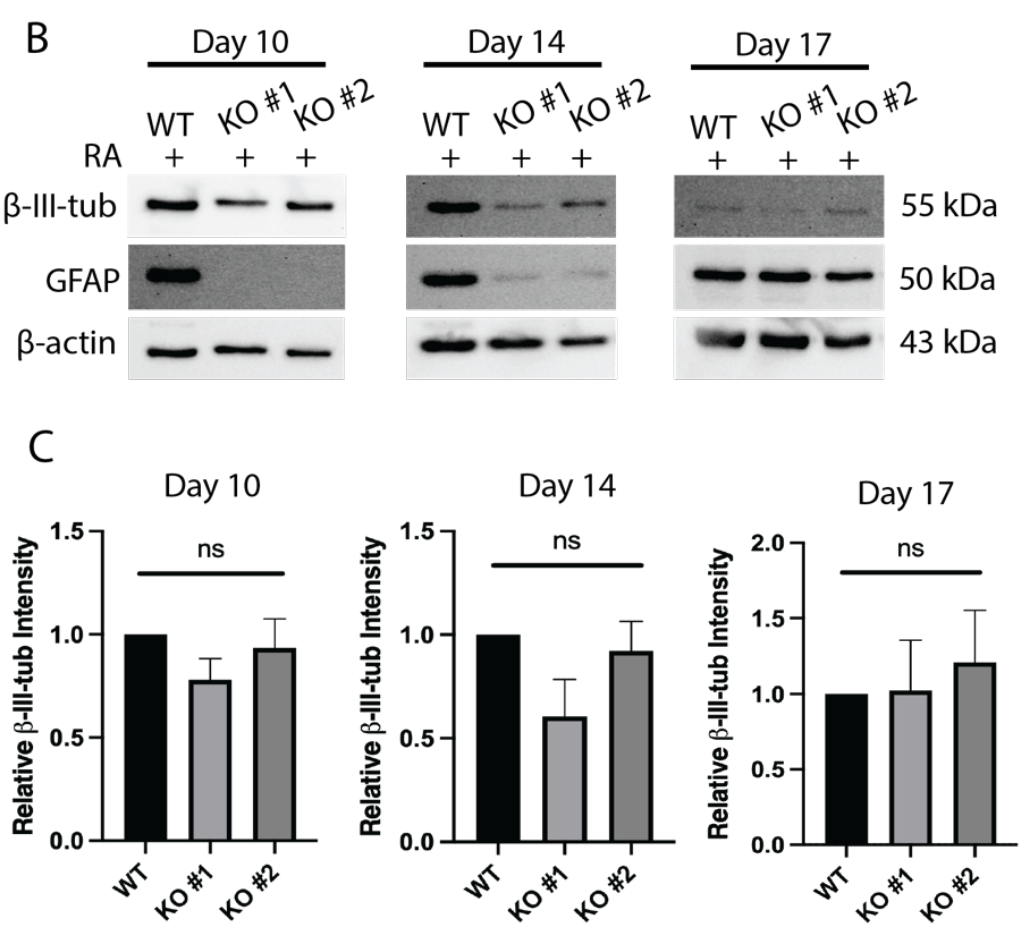

D

Day 10

Day 14

Day 17
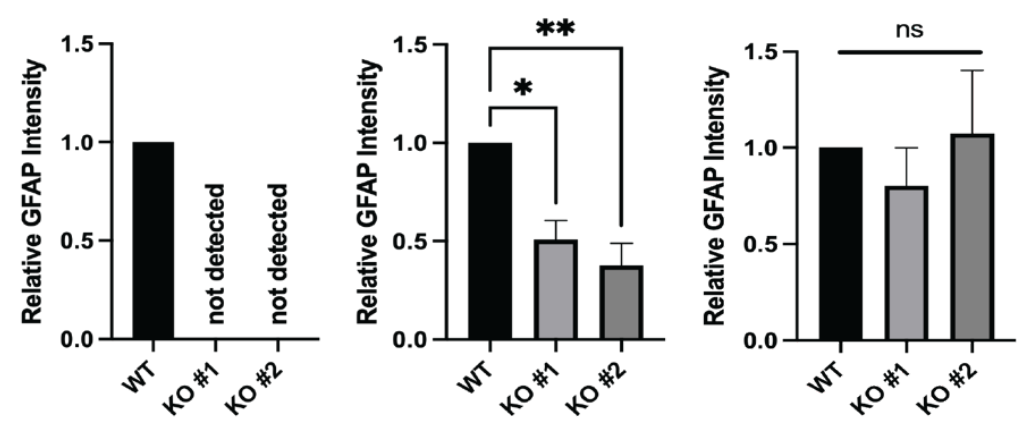

Figure 6 - Sufu ${ }^{-/}$delays astrocyte formation without affecting neuron differentiation. A) Immunoblot of neuron marker $\beta$-IIItubulin and astrocyte marker glial fibrillary acidic protein (GFAP) on days 0-17 in Sufu $u^{-/}$KO \#2 clone. B) Immunoblots of $\beta$-IIItubulin and GFAP on day 10, day 14 and day 17 in wildtype (WT) and Sufu- clones, and densitometric analyses of immunoblots of B) showing relative intensities of $\mathbf{C}) \beta$-III-tubulin and D) GFAP. $* P<0.05$, $* * P<0.01$. 


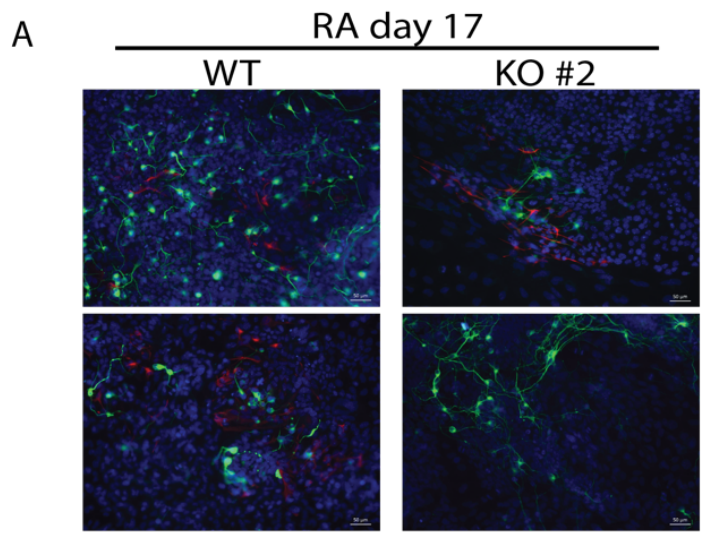

GFAP/ $\beta$-III-tubulin/DAPI

\section{B}
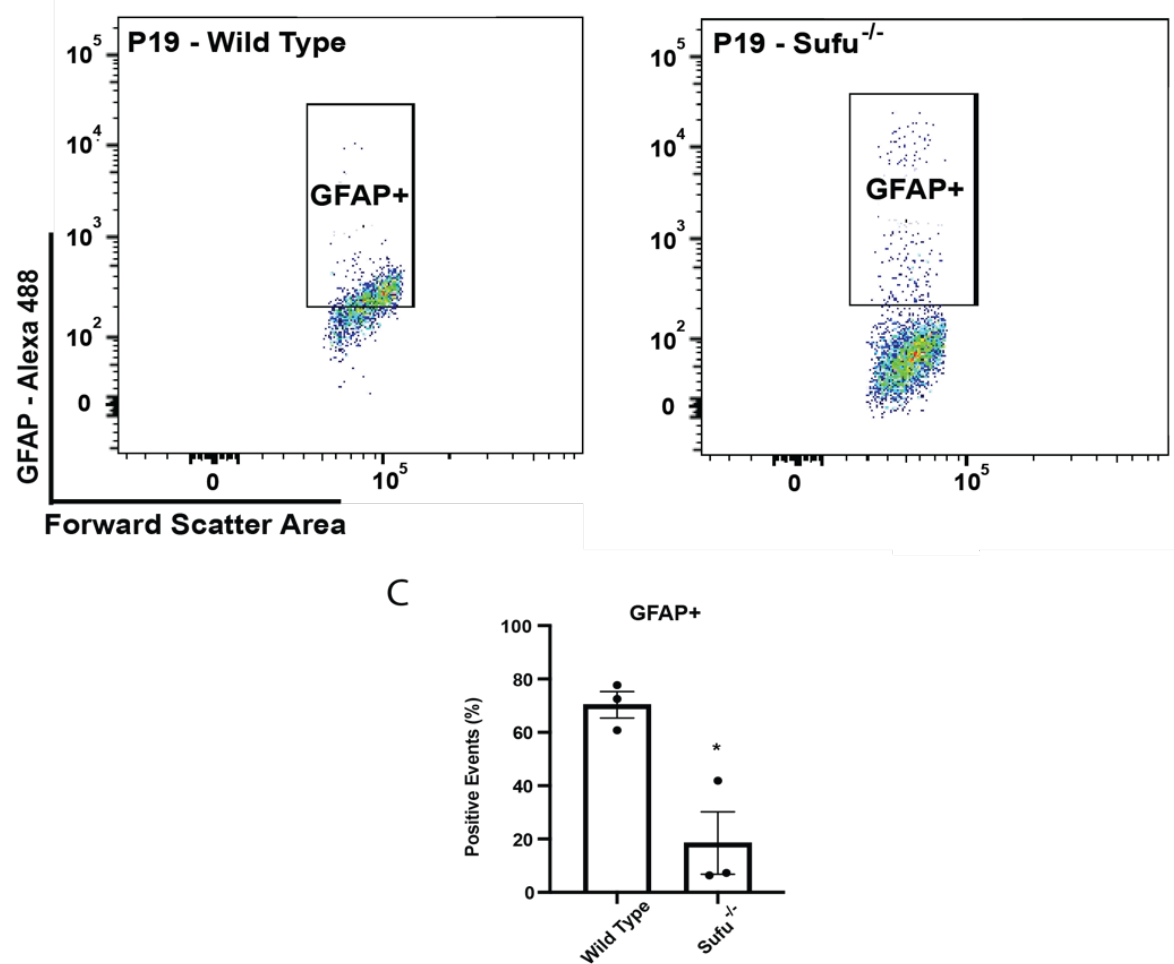

Figure 7 - Sufu $u^{-\leftarrow}$ decreases astrocyte differentiation. A) Astrocyte marker glial fibrillary acidic protein (GFAP) immunofluorescence (red), neuron marker $\beta$-III-tubulin (green) and nuclear DAPI (blue) in cells of wildtype (WT) and Sufu $u^{-/}$KO clone \#2 on day 17. B) Flow cytometry of GFAP-positive WT and $S u f u^{-/-}$KO \#2 cells on day 17 and quantified in $\mathbf{C}$. ${ }^{*}<0.05$.

\subsection{Sufu loss over-activates Hh signaling through loss of Gli3}

We previously determined that Sufu ${ }^{-/}$induced expression of Hh target genes in untreated, undifferentiated cultures (Figure 5D), and showed that the loss of this regulator delayed and decreased astrocyte differentiation (Figure 6 and Figure 7). Gli1 was also investigated to determine if its expression as a Hh target gene was affected in the Sufur cells. RT-qPCR results showed that Sufu- cells had significantly higher Gli1 expression compared to WT cells at day 0, 10, 14 and 17 of RA treatment (Figure 8A). Ptch1, another Hh target gene was explored, and like the Glil expression patten, it too showed significantly higher expression in Sufü- cells compared to WT cells treated with RA treatment (Figure 8B). Given previous work showing the connection between SUFU and GLI3 processing [7], we investigated Gli3 expression and protein levels. No significant differences were seen in Gli3 between Sufi-^ and WT cells (Figure 8C); however, at the protein level, the GLI3 repressor was not detected in Sufur cells (Figure 8D). More surprisingly, signals corresponding to the unprocessed GLI3 were also not detected (Figure 8D). Thus, the higher levels of expression of $\mathrm{Hh}$ target genes in Sufü ${ }^{-/}$cells would indicate that the Hh pathway was active, but since no significant changes to 
Gli3 were seen in either cell type, the loss of SUFU likely caused post-translational perturbations to the GLI3 protein. Nevertheless, the data support the notion that the delayed and decreased astrocyte differentiation seen in Sufu $u^{-/-}$cells is the result of the aberrant activation of Hh signaling and the loss of GLI3.

A

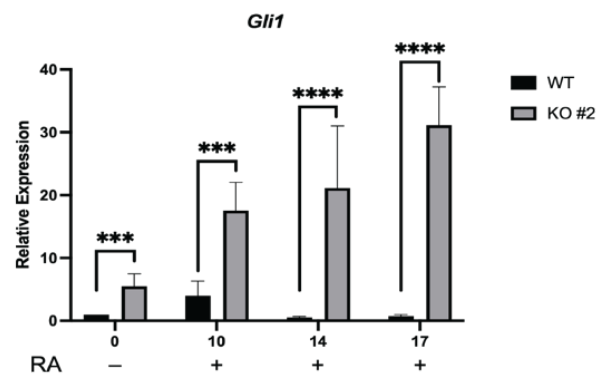

B

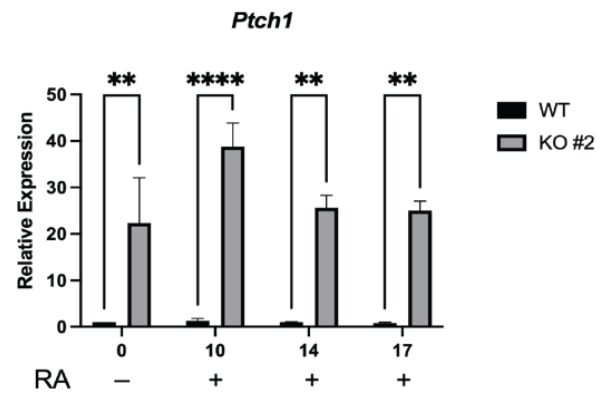

C
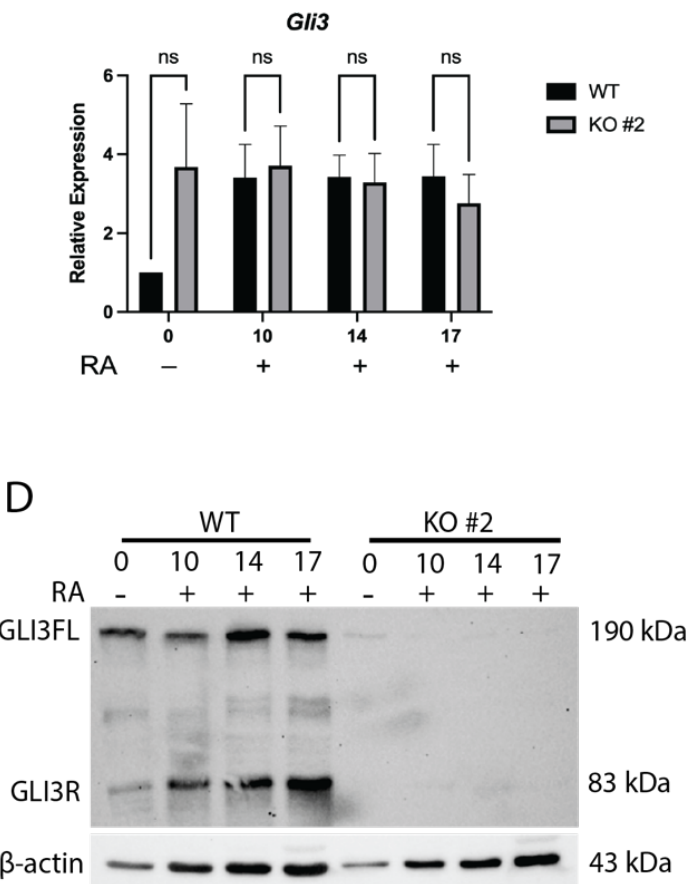

Figure 8 -Suf $\mathbf{~}^{-/-}$activates Hh signaling and causes the loss of Gli3. Expression of Hh target genes A) Glil and B) Ptchl and C) Gli3 encoding transcription factor in wildtype (WT) and $S u f u^{-1}$ KO \#2 cells on days 0-17. D) Immunoblot of GLI3 full length (GLI3FL) and GLI3 repressor (GLI3R) in WT and Sufü KO \#2 cells on days $0-17{ }^{*-} P<0.05,{ }^{*} P<0.01,{ }^{*} * *<0.001$, $* * * * P<0.0001$.

\section{Discussion}

Hh signaling is involved in neural tube closure and neural differentiation in vivo [as reviewed by 1,3 ] where the presence of Hh ligand induces a protein interaction cascade that results in the transcription of target genes through the GLI transcription factors $[3,7]$. Negative regulators of the pathway include the transmembrane receptor Patched and the cytoplasmic regulator SUFU [3]. The loss of these essential regulators results in medulloblastoma and Nevoid Basal Cell Carcinoma Syndrome [12-14, 25]; however, less is known about how the regulation of the pathway is involved in vertebrate neural development. Using the mouse P19 embryonal carcinoma cell model we determined that SUFU was essential for in vitro neural differentiation as its loss activates Hh signaling and affects not only the post-translational regulation of GLI3, but GLI3 itself. As important, this loss of SUFU resulted in the perturbed timing and proportion of astrocytes that formed. However, since SUFU loss had no apparent effect on neuronal differentiation this restricted regulation of the Hh pathway must be glial-specific.

\subsection{Timing of Hh signaling}

RA-induced neural differentiation of P19 cells is well established [18, 19, 26], but few studies have explored it and Hh signaling [20, 21, 27]. RA is known to act in these cells through the transcription factor FOXA1, which promotes the early expression of $S h$ [27], but the precise timing of pathway activation throughout differentiation has not been heretofore reported. We have shown that RA triggers Hh pathway activation early in neural specification (Figure 1 and Figure 2) and significantly, must remain inactive during later stages (Figure 3 and Figure 6). As in previous studies, we demonstrated that RA induced Shh expression, but Ihh and Dhh were also expressed (Figure 2). IHH and DHH, like SHH, can activate the pathway as evident in long bone differentiation [28, reviewed in 29] and gonad development [30-32], respectively. Similar results showing early transcriptional activation of all Hh 
ligands was reported with human embryonic stem cells induced to form neural tissues [33]. Thus, it is likely that SHH is responsible, but since transcripts encoding all ligands are induced by RA it remains unclear which isoform triggers pathway activation in the P19 model. Nevertheless, RA induction of a Hh ligand activates the pathway, but our evidence indicates that Hh signaling is inhibited during later stages of neural differentiation (Figure 3), coinciding with astrocyte specification. Furthermore, results strongly suggest that the pathway is temporally regulated to maintain it in an off configuration required for glial cell differentiation. Incidentally, previous Hh studies with P19 cells have only documented up to 8-10 days post RA-induction [20, 27], where the present work investigates beyond terminal neuron differentiation. Although we, like others, have demonstrated the presence of Hh signaling in P19 neural differentiation, other signaling pathways including those linked to Wnt, Fgf and Yap are also involved during early P19 cell specification [21, 34-36]. Considering our study, these other pathways require further investigation to determine if they too are temporally regulated to facilitate differentiation. However, since our focus was on the importance of this negative regulation on Hh signaling, specifically by SUFU, was further investigated.

\subsection{SUFU loss ectopically activates Hh target gene transcription}

SUFU is a tumor suppressor protein required for mouse embryogenesis [10, 12-14], but must be regulated for Hh signaling to occur. A previous report shows that SUFU levels are depleted through the ubiquitin-proteasome pathway following Hh signal activation [37]. Hh ligands are induced within 1 day of RA treatment (Figure 2) and given previous reports on SUFU regulation, we had expected an early decrease in SUFU levels that might increase to basal levels later during differentiation. Our results, however, showed no significant change in SUFU levels during early neural differentiation and there was a relative decrease later during astrocyte differentiation (Figure 4). The mechanism of SUFU depletion at these later stages was not determined, nor were SUFU post-translational modifications examined at any stage, but it should be noted that differential phosphorylation and ubiquitination can both promote or inhibit SUFU stability and/or GLI interaction [38-41]. Instead of further examining these post-translational modifications we decided to use CRISPR-Cas9 and genetically deplete SUFU from P19 cells. CRISPR-Cas9-mediated knockout of Sufu supported previous in vitro studies in both mouse and human embryonic stem cells that show its loss induces the ectopic transcription of Hh target genes (Figure 5 and Figure 8) [14, 33, 42, 43]. However, SUFU-deficient P19 cells did not differentiate in the absence of RA (Figure 6A) despite the previous report that Gli2 overexpression in P19 cells was sufficient to induce neuron differentiation under similar conditions [20]. Despite this discrepancy other avenues, including counting cell number and/or measuring the timing of differentiation, were examined.

\subsection{SUFU loss delays and decreases astrocyte differentiation}

Since Hh signaling plays key roles during many stages of vertebrate brain development [44, 45], the loss of SUFU or ectopic Hh signal activation is likely to affect neural differentiation. In support, when SUFU is knocked out in the embryo the mutation is embryonic lethal and the resulting phenotypes mimic Ptch $1^{-/}$mutants [10, 46]. Conditional knockouts of Sufu at various stages of brain development also results in decreased neuron differentiation and disorganized glial cell arrangements [15, 47], and in human embryonic stem cells there is an increase to neuroectodermal gene expression following SHH treatment [33]. In contrast, however, no change is seen in ectoderm differentiation following Sufu knockout in mouse ESCs [43]. Discrepancies also exist within the same cell type as evident in P19 cells where Gli2 overexpression induces neuron differentiation in the absence of RA [20], while Gli2 knockdown produces the opposite effect albeit under different differentiation conditions [21]. Although it is difficult to explain these differences, our work showed the knockout of Sufu was accompanied by the consistent over-activation of Hh target gene expression (Figure 5 and Figure 8), although Gli2 was not examined. There was, however no detectable change in neuron differentiation in SUFU-deficient cells (Figure 6), which is in support of the work by Hoelzl et al. [43], who found neuronal tissue derived from mouse embryonic stem cells depleted of SUFU. Furthermore, our results that showed a delay and decrease in glial cell differentiation with the loss of SUFU (Figure 6) may explain the disorganization of glial cells observed in the conditional Sufu knockouts described in mouse embryos [15, 47]. Overall, our research would indicate that Hh signaling is induced by RA, but the pathway must remain in the off state for proper astrocyte differentiation, and as described below the evidence points to SUFU regulation involves GLI3. 


\subsection{SUFU loss causes loss of Gli3}

Although we showed that the loss of SUFU ectopically activates the transcription of Hh target genes (Figure 5) and this is well supported $[14,33,42,43]$, the connection between aberrant glial cell differentiation and SUFU deficiency came from examining Hh target genes later during the differentiation stages. At these stages Gli1 and Ptch1, but not Gli3, expression was found to be higher when Sufu was knocked out (Figure8). However, previous reports with mouse show that SUFU is required for proper GLI3 processing [48] and its absence leads to reduced or absent GLI3 protein levels [11, 42]. Conditional Sufu knockouts in the mouse brain also show reduced or absent GLI3 protein $[15,16,47,49]$. Together these studies support our results showing the loss of GLI3 processing, and in fact of GLI3 in general (Figure 8) in the Sufu knock out lines is one reason why there may be decreased and/or delayed astrocyte formation.

\section{Conclusions}

We have reported the Hh signaling profile in P19 neurogenesis and demonstrated an essential role of a negative regulator of this pathway, SUFU, in maintaining the pathway in the off state to allow proper glial cell differentiation. We propose that the mechanism requires SUFU and, when absent, GLI3 is not processed into a repressor required to regulate the Hh pathway as seen in later stages when Sufu is intact. In fact, there is no full length GLI3 to be processed despite the Gli3 message being present in Sufu nulls. Thus, this study highlights the importance of the regulation of the Hh pathway by SUFU on neurogenesis and specifically the timing of differentiation of supporting glial cells.

Author Contributions: Conceptualization, D.M.S. and G.M.K.; methodology, D.M.S.; flow cytometry analysis, J.G.D.; formal analysis, G.M.K. and D.M.S.; writing, first draft-D.M.S.; writing — review and editing, D.M.S. and G.M.K.; supervision, G.M.K.; funding acquisition, G.M.K. All authors have read and agreed to the published version of the manuscript.

Funding: This research was funded by the Natural Sciences and Engineering Research Council (NSERC) of Canada, Discovery Grant R2615A02 to G.M.K. D.M.S. acknowledges support from the School of Graduate and Postdoctoral Studies, University of Western Ontario; the Collaborative Graduate Specialization in Developmental Biology, University of Western Ontario; the Children's Health Research Institute; and NSERC for a PGS D scholarship.

Acknowledgments: We would like to also thank members past and present of the Kelly lab for helpful discussions.

Conflicts of Interest: The authors declare no conflict of interest.

\section{References}

1. Wilson, L., \& Maden, M. (2005). The mechanisms of dorsoventral patterning in the vertebrate neural tube. Developmental Biology, 282 , 1-13. https://doi.org/10.1016/j.ydbio.2005.02.027

2. Dahmane, N., \& Ruiz, A. (1999). Sonic hedgehog and cerebellum development. Development, 3100, 3089-3100.

3. Briscoe, J., \& Thérond, P. P. (2013). The mechanisms of Hedgehog signalling and its roles in development and disease. Nature Reviews Molecular Cell Biology, 14(7), 418-431. https://doi.org/10.1038/nrm3598

4. Han, Y., Shi, Q., \& Jiang, J. (2015). Multisite interaction with Sufu regulates Ci/Gli activity through distinct mechanisms in Hh signal transduction. Proceedings of the National Academy of Sciences, 112(20), 6383-6388. https://doi.org/10.1073/pnas.1421628112

5. Tempe, D., Casas, M., Karaz, S., Blanchet-Tournier, M.-F., \& Concordet, J.-P. (2006). Multisite Protein Kinase A and Glycogen Synthase Kinase 3 Phosphorylation Leads to Gli3 Ubiquitination by SCF TrCP. Molecular and Cellular Biology, 26(11), 4316-4326. https://doi.org/10.1128/MCB.02183-05

6. Hui, C., \& Angers, S. (2011). Gli proteins in development and disease. Annual Review of Cell and Developmental Biology, 27(1), 513-537. https://doi.org/10.1146/annurev-cellbio-092910-154048

7. Humke, E. W., Dorn, K. V., Milenkovic, L., Scott, M. P., \& Rohatgi, R. (2010). The output of Hedgehog signaling is controlled by the dynamic association between Suppressor of Fused and the Gli proteins. Genes and Development, 24(7), 670-682. https://doi.org/10.1101/gad.1902910

8. Haycraft, C. J., Banizs, B., Aydin-Son, Y., Zhang, Q., Michaud, E. J., \& Yoder, B. K. (2005). Gli2 and Gli3 localize to cilia and require the intraflagellar transport protein Polaris for processing and function. PLoS Genetics, 1(4), e53. https://doi.org/10.1371/journal.pgen.0010053 
9. Svärd, J., Henricson, K. H., Persson-Lek, M., Rozell, B., Lauth, M., Bergström, Å., ... Teglund, S. (2006). Genetic elimination of suppressor of fused reveals an essential repressor function in the mammalian hedgehog signaling pathway. Developmental Cell, 10(2), $187-197$. https://doi.org/10.1016/j.devcel.2005.12.013

10. Cooper, A. F., Yu, K. P., Brueckner, M., Brailey, L. L., Johnson, L., McGrath, J. M., \& Bale, A. E. (2005). Cardiac and CNS defects in a mouse with targeted disruption of suppressor of fused. Development, 132(19), 4407-4417. https://doi.org/10.1242/dev.02021

11. Makino, S., Zhulyn, O., Mo, R., Puviindran, V., Zhang, X., Murata, T., ... Gondo, Y. (2015). T396I mutation of mouse Sufu reduces the stability and activity of Gli3 repressor. PLoS ONE, 10(3), 1-15. https://doi.org/10.1371/journal.pone.0119455

12. Taylor, M. D., Liu, L., Raffel, C., Hui, C. chung, Mainprize, T. G., Zhang, X., ... Hogg, D. (2002). Mutations in SUFU predispose to medulloblastoma. Nature Genetics, 31(3), 306-310. https://doi.org/10.1038/ng916

13. Smith, M. J., Beetz, C., Williams, S. G., Bhaskar, S. S., Sullivan, J. O., Anderson, B., .. William, G. (2018). Germline mutations in SUFU cause Gorlin Syndrome - Associated Childhood Medulloblastoma and redefine the risk associated with PTCH1 mutations. Journal of Clinical Oncology, 32(36), 4155-4161. https://doi.org/10.1200/JCO.2014.58.2569

14. Urman, N. M., Mirza, A., Atwood, S. X., Whitson, R. J., Sarin, K. Y., Tang, J. Y., \& Oro, A. E. (2016). Tumor-derived suppressor of fused mutations reveal hedgehog pathway interactions. PLoS ONE, 11(12), 1-10. https://doi.org/10.1371/journal.pone.0168031

15. Kim, J. J., Jiwani, T., Erwood, S., Loree, J., \& Rosenblum, N. D. (2018). Suppressor of fused controls cerebellar neuronal differentiation in a manner modulated by GLI3 repressor and Fgf15. Developmental Dynamics, 247(1), 156-169. https://doi.org/10.1002/dvdy.24526

16. Kim, J. J., Gill, P. S., Rotin, L., van Eede, M., Henkelman, R. M., Hui, C.-C., \& Rosenblum, N. D. (2011). Suppressor of Fused controls midhindbrain patterning and cerebellar morphogenesis via GLI3 repressor. Journal of Neuroscience, 31(5), $1825-1836$. https://doi.org/10.1523/JNEUROSCI.2166-10.2011

17. Mcburney, M. (1993). P19 embryonal carcinoma cells. International Journal of Developmental Biology, 37, $135-140$.

18. McBurney, M. W., Jones-Villeneuve, E. M. V., Edwards, M. K. S., \& Anderson, P. J. (1982). Control of muscle and neuronal differentiation in a cultured embryonal carcinoma cell line. Nature, 299(5879), 165-167. https://doi.org/10.1038/299165a0

19. McBurney, M. W., Reuhl, K. R., Ally, a I., Nasipuri, S., Bell, J. C., \& Craig, J. (1988). Differentiation and maturation of embryonal carcinomaderived neurons in cell culture. The Journal of Neuroscience, 8(3), 1063-73. Retrieved from http://www.ncbi.nlm.nih.gov/pubmed/2894413

20. Voronova, A., Fischer, A., Ryan, T., Al Madhoun, A., \& Skerjanc, I. S. (2011). Ascl1/Mash1 is a novel target of Gli2 during Gli2-induced neurogenesis in P19 EC cells. PLoS ONE, 6(4). https://doi.org/10.1371/journal.pone.0019174

21. Lin, Y. T., Ding, J. Y., Li, M. Y., Yeh, T. S., Wang, T. W., \& Yu, J. Y. (2012). YAP regulates neuronal differentiation through Sonic hedgehog signaling pathway. Experimental Cell Research, 318(15), 1877-1888. https://doi.org/10.1016/j.yexcr.2012.05.005

22. Ran, F. A., Hsu, P. D., Wright, J., Agarwala, V., Scott, D. A., \& Zhang, F. (2013). Genome engineering using CRISPR-Cas9 system. Nature Protocols, 8(11), 2281-2308. https://doi.org/10.1007/978-1-4939-1862-1_10

23. Brinkman, E. K., Chen, T., Amendola, M., \& Van Steensel, B. (2014). Easy quantitative assessment of genome editing by sequence trace decomposition. Nucleic Acids Research, 42(22), 1-8. https://doi.org/10.1093/nar/gku936

24. Dunaeva, M., Michelson, P., Kogerman, P., \& Toftgard, R. (2003). Characterization of the physical interaction of Gli proteins with SUFU proteins. Journal of Biological Chemistry, 278(7), 5116-5122. https://doi.org/10.1074/jbc.M209492200

25. Hahn, H., Wicking, C., Zaphiropoulos, P. G., Gailani, M. R., Shanley, S., Chidambaram, A., ... Bale, A. E. (1996). Mutations of the human homolog of drosophila patched in the nevoid basal cell carcinoma syndrome. Cell, 85(6), 841-851. https://doi.org/10.1016/S0092-8674(00)812684

26. Jones-Villeneuve, E. M., McBurney, M. W., Rogers, K. A., \& Kalnins, V. I. (1982). Retinoic acid induces embryonal carcinoma cells to differentiate into neurons and glial cells. The Journal of Cell Biology, 94(2), 253-262. https://doi.org/10.1083/jcb.94.2.253

27. Tan, Y., Xie, Z., Ding, M., Wang, Z., Yu, Q., Meng, L., ... Chen, Y. (2010). Increased levels of FoxA1 transcription factor in pluripotent P19 embryonal carcinoma cells stimulate neural differentiation. Stem Cells and Development, 19(9), 1365-1374. https://doi.org/10.1089/scd.2009.0386

28. St-Jacques, B., Hammerschmidt, M., \& McMahon, A. P. (1999). Indian hedgehog signaling regulates proliferation and differentiation of chondrocytes and is essential for bone formation. Genes and Development, 13(16), 2072-2086. https://doi.org/10.1101/gad.13.16.2072

29. Bechtold, T. E., Koyama, E., Kurio, N., Nah, H. D., Saunders, C., \& Billings, P. C. (2019). The roles of Indian hedgehog signaling in TMJ formation. International Journal of Molecular Sciences, 20(24). https://doi.org/10.3390/ijms20246300 
30. Bashamboo, A., \& McElreavey, K. (2015). Human sex-determination and disorders of sex-development (DSD). Seminars in Cell and Developmental Biology, 45, 77-83. https://doi.org/10.1016/j.semcdb.2015.10.030

31. Bitgood, M. J., Shen, L., \& McMahon, A. P. (1996). Sertoli cell signaling by Desert hedgehog regulates the male germline. Current Biology, 6(3), 298-304. https://doi.org/10.1016/S0960-9822(02)00480-3

32. Clark, A. M., Garland, K. K., \& Russell, L. D. (2000). Desert hedgehog (Dhh) gene is required in the mouse testis for formation of adult-type Leydig cells and normal development of peritubular cells and seminiferous tubules. Biology of Reproduction, 63(6), 1825-1838. https://doi.org/10.1095/biolreprod63.6.1825

33. Wu, S. M., Choo, A. B. H., Yap, M. G. S., \& Chan, K. K. K. (2010). Role of Sonic hedgehog signaling and the expression of its components in human embryonic stem cells. Stem Cell Research, 4(1), 38-49. https://doi.org/10.1016/j.scr.2009.09.002

34. Tang, K., Yang, J., Gao, X., Wang, C., Liu, L., Kitani, H., ... Jing, N. (2002). Wnt-1 promotes neuronal differentiation and inhibits gliogenesis in P19 cells. Biochemical and Biophysical Research Communications, 293(1), 167-173. https://doi.org/10.1016/S0006-291X(02)00215-2

35. Jing, X. T., Wu, H. T., Wu, Y., Ma, X., Liu, S. H., Wu, Y. R., ... Fan, M. (2009). DIXDC1 promotes retinoic acid-induced neuronal differentiation and inhibits gliogenesis in P19 cells. Cellular and Molecular Neurobiology, 29(1), 55-67. https://doi.org/10.1007/s10571-008-9295-9

36. Chen, W., Caihong, X., Wei, B., Li, L., Lin, W., Chen, Y.-G., ... Jing, N. (2006). Cell aggregation-induced FGF8 elevation Is essential for P19 cell neural differentiation. Molecular Biology of the Cell, 17(July), 3075-3084. https://doi.org/10.1091/mbc.e05-11-1087

37. Yue, S., Chen, Y., \& Cheng, S. Y. (2009). Hedgehog signaling promotes the degradation of tumor suppressor Sufu through the ubiquitinproteasome pathway. Oncogene, 28(4), 492-499. https://doi.org/10.1038/onc.2008.403

38. Takenaka, K., Kise, Y., \& Miki, H. (2007). GSK3 $\beta$ positively regulates Hedgehog signaling through Sufu in mammalian cells. Biochemical and Biophysical Research Communications, 353(2), 501-508. https://doi.org/10.1016/j.bbrc.2006.12.058

39. Infante, P., Faedda, R., Bernardi, F., Bufalieri, F., Severini, L. L., Alfonsi, R., ... Di Marcotullio, L. (2018). Itch/ $\beta$-Arrestin2-dependent nonproteolytic ubiquitylation of $\mathrm{SuFu}$ controls Hedgehog signalling and medulloblastoma tumorigenesis. Nature Communications, 9(1), 1-17. https://doi.org/10.1038/s41467-018-03339-0

40. Chen, Y., Yue, S., Xie, L., Pu, X. H., Jin, T., \& Cheng, S. Y. (2011). Dual phosphorylation of suppressor of fused (Sufu) by PKA and GSK3 $\beta$ regulates its stability and localization in the primary cilium. Journal of Biological Chemistry, 286(15), 13502-13511. https://doi.org/10.1074/jbc.M110.217604

41. Wang, Y., Li, Y., Hu, G., Huang, X., Rao, H., Xiong, X., ... Luo, S. (2016). Nek2A phosphorylates and stabilizes SuFu: A new strategy of Gli2/Hedgehog signaling regulatory mechanism. Cellular Signalling, 28(9), 1304-1313. https://doi.org/10.1016/j.cellsig.2016.06.010

42. Chen, M. H., Wilson, C. W., Li, Y. J., Law, K. K. Lo, Lu, C. S., Gacayan, R., ... Chuang, P. T. (2009). Cilium-independent regulation of Gli protein function by Sufu in Hedgehog signaling is evolutionarily conserved. Genes and Development, 23(16), 1910-1928. https://doi.org/10.1101/gad.1794109

43. Hoelzl, M. A., Heby-Henricson, K., Bilousova, G., Rozell, B., Kuiper, R. V, Kasper, M., ... Teglund, S. (2015). Suppressor of Fused plays an important role in regulating mesodermal differentiation of murine embryonic stem cells In vivo. Stem Cells and Development, 24(21), 2547-2560. https://doi.org/10.1089/scd.2015.0050

44. Memi, F., Zecevic, N., \& Radonjić, N. (2018). Multiple roles of Sonic Hedgehog in the developing human cortex are suggested by its widespread distribution. Brain Structure and Function, 223(5), 2361-2375. https://doi.org/10.1007/s00429-018-1621-5

45. Belgacem, Y. H., Hamilton, A. M., Shim, S., Spencer, K. A., \& Borodinsky, L. N. (2016). The many hats of Sonic hedgehog signaling in nervous system development and disease. Journal of Developmental Biology, 4(4). https://doi.org/10.3390/jdb4040035

46. Goodrich, L. V., Milenković, L., Higgins, K. M., \& Scott, M. P. (1997). Altered neural cell fates and medulloblastoma in mouse patched mutants. Science, 277(5329), 1109-1113. https://doi.org/10.1126/science.277.5329.1109

47. Yabut, O. R., Fernandez, G., Yoon, K., Pleasure, S. J., Yabut, O. R., Fernandez, G., ... Pleasure, S. J. (2015). Suppressor of Fused Is critical for maintenance of neuronal progenitor identity during corticogenesis. Cell Reports, 12(12), 2021-2034. https://doi.org/10.1016/j.celrep.2015.08.031

48. Kise, Y., Morinaka, A., Teglund, S., \& Miki, H. (2009). Sufu recruits GSK3 $\beta$ for efficient processing of Gli3. Biochemical and Biophysical Research Communications, 387(3), 569-574. https://doi.org/10.1016/j.bbrc.2009.07.087

49. Jiwani, T., Kim, J. J., \& Rosenblum, N. D. (2020). Suppressor of fused controls cerebellum granule cell proliferation by suppressing Fgf8 and spatially regulating Gli proteins. Development, 147(3), 1-17. https://doi.org/10.1242/dev.170274 
bioRxiv preprint doi: https://doi.org/10.1101/2021.08.24.457497; this version posted August 24, 2021. The copyright holder for this preprint (which was not certified by peer review) is the author/funder. All rights reserved. No reuse allowed without permission. 